\title{
Tracking haplotype for QTLs associated with Fusarium head blight resistance in Japanese wheat (Triticum aestivum L.) lineage
}

Sayaka Niwa', Yusuke Kazama ${ }^{1,2}$, Tomoko Abe $^{2}$ and Tomohiro Ban ${ }^{1 *}$

\begin{abstract}
Background: Fusarium head blight (FHB) resistance in wheat (Triticum aestivum L.) has been enhanced by combining resistant alleles with transgressive segregation. In Japan, intensive FHB resistance breeding was initiated in 1970s in Kyushu and in 1990s in Hokkaido. The breeding objectives for climatic adaptation are different between Kyushu and Hokkaido. The objective of this study is to determine how and what type of alleles should be introduced into current cultivars by characterizing the track of allelic selection in both breeding lineages.

Results: Herein, we traced haplotype of SSR markers for allelic selection of FHB resistance-related quantitative trait loci (QTLs) in the Japanese breeding lineages of Kyushu and Hokkaido where the climatic adaptations of wheat are dissimilar. In Kyushu, resistant haplotypes on chromosomes 3BS, 5AS, and 6BS were frequently found in old cultivars, and those on $3 \mathrm{BS}$ and $5 \mathrm{AS}$ were transmitted to modern cultivars with incorporating the resistant allele on 2DL. The winter cultivars of Hokkaido lacked the resistant allele set of the markers at 3BS, but the resistant haplotypes at 2DL and 5AS were predominantly retained. The resistant allele combinations at 6BS and 2DS were mostly excluded from Kyushu and the winter cultivars of Hokkaido. The susceptible haplotype on 2DS was co-inherited with the semi-dwarfing allele at Xgwm261 which is diagnostic for the presence of the dwarfing gene Rht8 and the allele of the photoperiod insensitive at Ppd-D1 in Kyushu, indicating a linkage drag between a FHB resistance allele and ones underling agronomic characters. In Hokkaido, six cultivars were found to have the resistant haplotype on 2DS with the semi-dwarfing allele of Xgwm261.
\end{abstract}

Conclusions: Our results suggest that a trade-off between FHB resistance and quality or agronomic traits has contributed to the history of Japanese FHB resistance breeding. To enhance FHB resistance in current cultivars, introducing the resistant allele of QTL on 2DS should be a promising option. The six winter cultivars found in the Hokkaido lineage can be used as new donors for introducing FHB resistance alleles of QTL on 2DS into modern cultivars together with the semi-dwarfing allele at Xgwm261.

Keywords: Fusarium head blight, Wheat, Breeding lineage, Allelic selection

\section{Background}

Fusarium head blight (FHB), caused by more than 17 species in the genus Fusarium, is a serious disease affecting small-grain crops, such as bread wheat (Triticum aestivum L.), durum wheat (Triticum turgidum L. subsp. durum), barley (Hordeum vulgare L.), and oat (Avena

\footnotetext{
*Correspondence: tban@yokohama-cu.ac.jp

${ }^{1}$ Kihara Institute for Biological Research, Yokohama City University, 641-12

Maioka, Totsuka, Yokohama, Kanagawa 244-0813, Japan

Full list of author information is available at the end of the article
}

sativa L.). FHB inflicts critical yield losses and contaminates food with mycotoxins, such as the trichothecene deoxynivalenol (DON). The Food and Agriculture Organization has set guidelines for the maximum acceptable limits of DON contamination [1]. The use of resistant cultivars in combination with agronomical control methods such as fungicide application and crop rotation has effectively reduced the impact of FHB [2]. Two types of phenotypic response have been characterized, resistance to the initial infection (type I) and resistance to spreading 
within a spike (type II) [3]. These FHB resistance traits in wheat are controlled by numerous quantitative trait loci (QTLs) [4, 5].

FHB-resistant Chinese wheat cultivar 'Sumai 3' and its derivatives have been globally utilized to breed FHBresistant cultivars [6]. 'Sumai 3' derivatives, such as the Shanghai and Wuhan series developed in China, have been used as the major sources of resistance alleles for the global wheat breeding programme of the International Maize and Wheat Improvement Center [7]. In quantitative genetic research, 'Sumai 3' has been used to identify QTLs related to FHB resistance. FHB resistance-related QTLs in 'Sumai 3' are located on chromosomes 2DL and 5AS (Qfhs.ifa-5A) for type I resistance, 3BS (Fhb1) for type II resistance, and 6BS (Fhb2) for field resistance as assessed by disease index scored in the field [8-16]. Recently, Fhb1 has been cloned and identified as a pore-forming toxin-like gene, demonstrating that 'Sumai 3' has the durable gene resistant to Fusarium species on 3BS [17]. 'Sumai 3' also has a susceptible allele for FHB at a QTL on chromosome 2DS [18, 19]. In a study that used a doubled-haploid population derived from a cross between 'Sumai 3' and the susceptible cultivar 'Gamenya', susceptible alleles in 'Sumai 3' and resistant alleles in 'Gamenya' on 2DS were detected. A candidate gene responsible for the FHB resistance on this region, TaMRP-D1, was also identified which encoded a multidrug resistance-associated protein [19].

In Japan, the main wheat production areas are Hokkaido and Kyushu, located in the northern and southwestern parts of the country, respectively. In Kyushu, the greatest FHB damage to wheat production occurred in 1963, and the development of FHB-resistant cultivars has since been a major priority. The Japanese cultivar 'Shinchunaga' was a useful crossing parent for FHB resistance in breeding programmes aimed at developing cultivars with stable, moderate resistance in the 1970s [20]. In 1976, 'Sumai 3' was introduced in Japan as a high-FHB resistance source [21]; several cultivars and parental lines descending from this parent cultivar have been developed, e.g. the resistant cultivars 'Norin PL-4' [22], 'Saikai 165' [23], 'Norin PL-9' [24], and the cultivar with a relatively low DON content 'Towaizumi' [25]. FHB resistance and DON accumulation of several cultivars as stated above have been extensively evaluated by Kubo and Kawada [26]. In Hokkaido, breeding efforts have historically focused on leaf rust resistance and cold tolerance. The FHB-damaged area in Hokkaido is relatively small compared to that of the Kyushu region in southwestern Japan owing to its relatively lower precipitation and humidity during wheat flowering and maturation. In 1996, after heavy rainfall, FHB strongly affected wheat production area in Hokkaido [27]. Nishio et al. [28] reported varietal differences in FHB resistance in winter wheat of Hokkaido and concluded that susceptibility was higher than that of the Kyushu and Tokai regions. Therefore, the breeding of FHB resistance cultivars is currently among the most important priorities in the region. 'Sumai 3' and 'Saikai 165' were introduced from the breeding programme in Kyushu as FHB-resistant parents to improve local cultivars with respect to FHB resistance in Hokkaido [27].

Considering that the breeding objectives are different between Kyushu and Hokkaido, favourable transmitted haplotypes could be affected by their objectives. To test this hypothesis and determine the important alleles for FHB resistance as well as to gain insight of the haplotypes that can facilitate further resistant breeding, we tracked haplotypes of the FHB resistance-related QTLs on chromosomes 2DS, 2DL, 3BS, 5AS, and 6BS in the released cultivars and breeding lines from the Kyushu and Hokkaido lineages.

\section{Methods \\ Plant material}

A total of 89 wheat cultivars predominantly developed and released in the Kyushu and Hokkaido regions were genotyped in this study (Additional file 1: Table S1). The seeds were sown on petri dishes and refrigerated at $4{ }^{\circ} \mathrm{C}$ for 4 days to break down seed dormancy. The petri dishes were transferred to a $25{ }^{\circ} \mathrm{C}$ chamber until germination. The germinated plants were transplanted to vinyl pots and grown in a glasshouse at $25{ }^{\circ} \mathrm{C}$. Leaf tips were collected at 18 days after sowing and stored at $-80^{\circ} \mathrm{C}$ for DNA extraction.

\section{Genotyping of haplotypes for wheat cultivars from the Kyushu and Hokkaido lineages}

DNA of each cultivar was extracted from the leaf tip of at least five individual plants using the DNeasy Plant Mini Kit (Qiagen, Venlo, Netherlands). Fewer than five individual plants were sampled for the following cultivars owing to low germination rates in their stocks: four individual plants in 'Tohoku 118,' 'Norin 58, 'Norin 24, 'U-11', 'Minturki', 'Kitami 19', and 'Kitami 35', three individual plants in 'Norin 8,' 'Norin 27, 'Norin 33', and 'Akasabishirazu 1', two individual plants in 'Hokuei', 'Kitami 16', and 'Kitami 18', and one individual plant in 'Hokkai 240'. DNA markers associated with FHB resistance-related QTLs on chromosomes 2DS, 2DL, 3BS, 5AS, and 6BS determined in 'Sumai 3' were used to genotype the haplotypes (Additional file 2: Table S2). Among the four markers associated with FHB resistance-related QTL on 5AS, two (Xgwm293 and Xgwm304) were polymorphic in 'Sumai 3' accessions ('Sumai 3-JPNy' and 'Sumai 3-AUT'); 'Sumai 3-JPNy' and 'Sumai 3-AUT' alleles were susceptible and 
resistant, respectively [29]. The Xgwm261 marker was used to detect genotypes associated with dwarfism as described previously [30]. The PCR products of UMN10 were analysed by sequencing with the Big Dye Terminator v. 3.1 Cycle Sequencing Kit (Applied Biosystems, Foster City, USA) and a 3730xl DNA Analyser (Applied Biosystems) using the same PCR primers. The PCR products of the other markers were electrophoresed using MultiNA (Shimadzu, Kyoto, Japan). Genotypes were classified by following the criteria outlined in Fig. 1. The genotype of $P p d-D 1$, a locus controlling photoperiod insensitivity on chromosome 2DS, was determined using a method previously described by Beales et al. [31], and its PCR product was electrophoresed using the QIAxcel Advanced System (Qiagen).

\section{Results}

Eighty-nine wheat cultivars mainly from two Japanese lineages, Kyushu and Hokkaido, were genotyped for their critical haplotypes to determine the distribution of FHB resistance-related QTL alleles identified in 'Sumai 3 ' as well as the $X g w m 261$ alleles associated with Rht8. In addition, we tracked genotypes related to the photoperiod-sensitivity locus $P p d-D 1$ to assess the possibility of linkage (i.e. linkage disequilibrium) with $P p d-D 1$ on chromosome 2DS. Photoperiod sensitivity (and specifically earliness) is an important trait for adaptation not only to avoid damage from FHB, but also to avoid preharvest sprouting in Japan. We also tracked genotypes related to the photoperiod-sensitivity locus $P p d-D 1$. Fourteen out of 19 markers on five chromosome regions tested in this study were polymorphic in 'Sumai 3-JPNy', 'Sumai 3-AUT', 'Gamenya', and 'Chinese Spring' (CS) plants (Fig. 1). The following haplotypes were identified: (1) 'Sumai 3-JPNy', (2) 'Sumai 3-AUT', (3) 'Gamenya', alleles specific to particular 'Gamenya' genotypes but also found in 'Sumai 3' accessions, and (4) 'CS', genotypespecific to 'CS' alleles, common alleles for the two 'Sumai
3' accessions. On chromosome 2DS, three haplotypes ('Sumai 3-AUT, 'Sumai 3-JPNy', and 'Gamenya') were determined (Fig. 1). Although a common haplotype was observed in 'Sumai 3-JPNy' and 'CS' plants, we termed it the 'Sumai 3-JPNy' haplotype. All studied cultivars were genotyped and compared based on their genotypes and haplotypes.

On chromosome 3BS of the Kyushu lineage, most cultivars had identical haplotype for the resistant 'Sumai 3' allele, while 'Kounosu 26', 'California', and 'Saitama 29' had the susceptible 'Gamenya' allele (Fig. 2). On chromosome 3BS, the susceptible haplotype was observed in 'Saitama 29' and absent in 'Eshimashinriki' and 'Kinki 35' tended to be replaced by the 'Sumai 3' allele from 'Shinchunaga' in their progeny (Fig. 2). The resistant haplotype from 'Shinchunaga', which arose in the 1930s, was inherited in 'Wheat Norin PL-9' plants bred in the 2010s. Resistant alleles combination of Xgwm293 and Xgwm304 on chromosome 5AS for 'Sumai 3-AUT' was found in 26 out of 38 cultivars tested (Fig. 2). The 'Sumai 3' haplotype on chromosome 6BS was found in 'Shinchunaga, 'Norin 20,' 'Norin 61', and 'Tokai 62' (Fig. 2). 'Shinchunaga' bred in the 1930s transmitted the haplotype to its descendants ('Norin 20' and 'Norin 61'). In one of the descendants bred 'Norin 26' in the 1930s, the 'Sumai 3' haplotype was not inherited from its parent 'Shinchunaga', but in progeny of 'Norin 26', Tokai series cultivars developed in 1960-1970s, the 'Sumai 3' haplotype was transmitted from 'Shinchunaga' (Fig. 2). After 'Shirasagikomugi' was bred in the 1950s, the 'Gamenya' haplotype (possibly derived from 'Kinki 35') was inherited by its progeny until the 2010s. The resistant haplotype of 'Sumai 3' on chromosome 2DL was retained after 'Hiyokukomugi' was bred in the 1960s. The resistant haplotype might have originated in 'Fukuokakomugi 18', if it was heterozygous in 'Saikai 95' (Fig. 3). On chromosome 2DS, two haplotypes ('Sumai 3-JPNy' and 'Gamenya') were frequent (Fig. 3). Old cultivars, 'Norin 5, 'Eshimashinriki,' 'Norin

Sumai 3-AUT Sumai 3-JPNy
3BS (UMN10)
5AS (Xbarc180, Xgwm293, and Xgwm304)
6BS (Xwmc398 and Xgwm644)
2DS (Xgwm261, Xwmc503, J06R, TaMRP-D1, J06F, Xwmo112, and Ppd-D1), 2DL (Xgwm539)
Fig. 1 Criteria for genotyping based on polymorphisms between control cultivars. Five alleles were defined: (1) 'Sumai 3-JPNy', (2) 'Sumai 3-AUT', (3)
'Sumai 3', common alleles for 'Sumai 3'accessions, (4) 'Gamenya', alleles specific for 'Gamenya' genotypes but also found in 'Sumai 3' accessions, and
(5) 'Chinese Spring', genotype-specific alleles




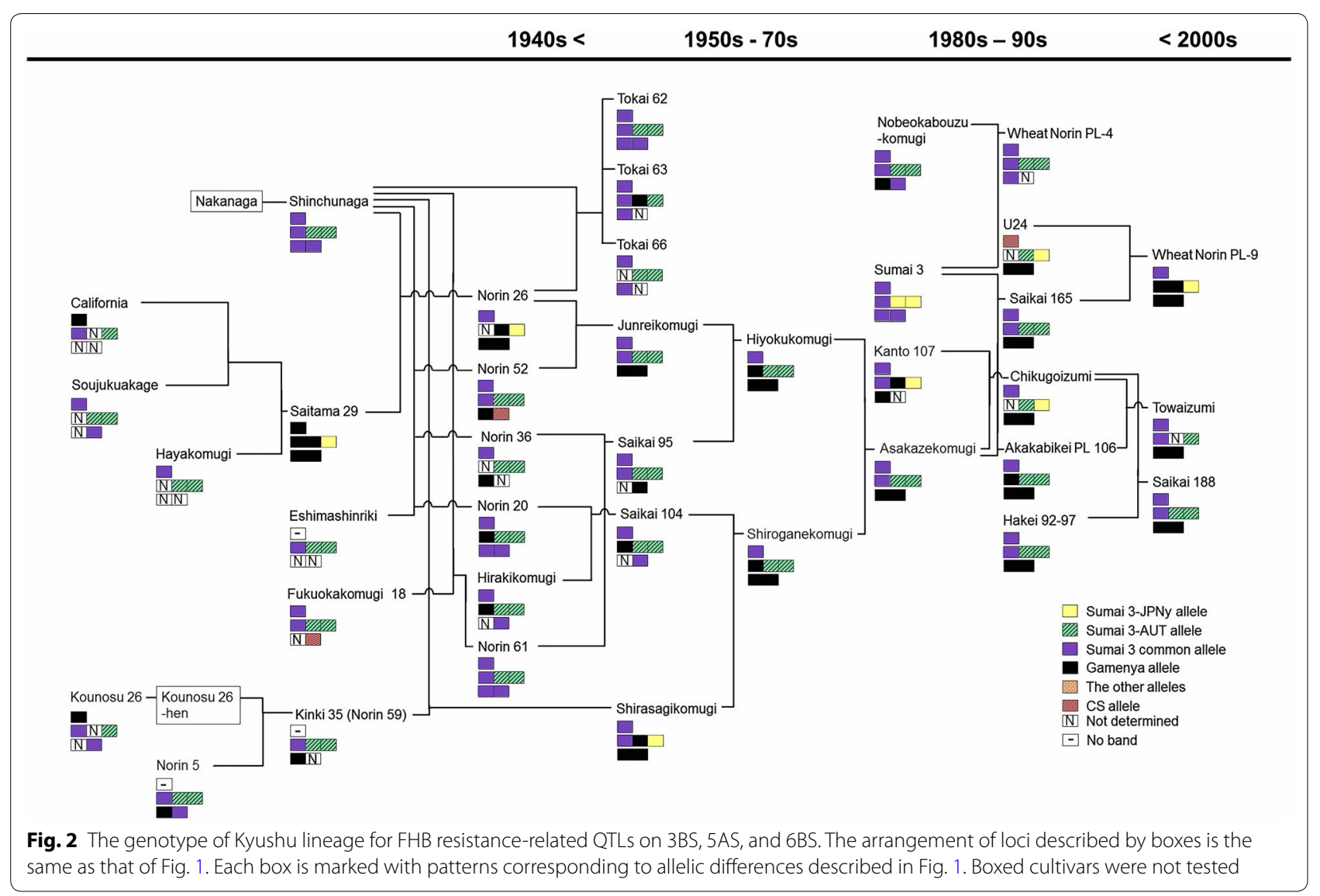

20', 'Kinki 35', and 'Saikai 104', carried the 'Gamenya' haplotype, with the oldest origins of the haplotype found in 'Eshimashinriki' and 'Kinki 35. The 'Gamenya' haplotype of 'Eshimashinriki' was inherited and selected in the breeding of 'Norin 20' and 'Saikai 104'. When 'Shiroganekomugi' was developed, the 'Gamenya' haplotype was replaced by 'Sumai 3-JPNy', originally derived from 'Shinchunaga', and it was eventually fixed in its descendants (Fig. 3). The prevalence of the semi-dwarfing allele for Rht8 confirmed with diagnostic SSR marker Xgwm261 increased among genotypes with the progression of selection after the 1970s (Fig. 3, see "Discussion" section).

In the Hokkaido lineage, both winter and spring wheat cultivars have been developed. In the Hokkaido lineage, some cultivars ('Norin 33', 'Norin 27', 'Norin 58, 'Norin 24,' 'Tohoku 103', 'Nanbukomugi', and 'Tohoku 118 ') developed in the Tohoku area were used as parent lines. In the winter cultivars, on chromosome 3BS, the resistant 'Sumai 3' haplotype was observed only in '14006', and the other cultivars harbouring this allele ('Norin 24' and 'Tokai 64') were introduced from outside of Hokkaido. The susceptible 'Gamenya' haplotype was initially harboured by some old cultivars including 'Tohoku 103' and 'Minturki' and transmitted to modern cultivars ('Kitahonami' and 'Yumechikara') bred in the 2000s (Fig. 4). 'Sumai 3-AUT' haplotype of Xgwm304 and Xgwm293 on 5AS was observed in 18 out of 36 tested cultivars (Fig. 4). The resistant 'Sumai 3-AUT' alleles Xgwm304 and Xgwm293 on 5AS from 'Akasabishirazu 1' bred in the 1920s were continuously inherited (through 'Hokuei', 'Kitami 19', 'Chihokukomugi,' 'Hokushin', and '13090') sequentially over an extended period of time (Fig. 4). The resistant 'Sumai 3' alleles at 6BS were detected at a minimum, but it was unlikely to be retained in progenies. The common 'Sumai 3' resistant haplotype on chromosome 2DL which initially found in foreign cultivars 'Hope' and 'Minturki' was not transmitted to their progeny. However, the 'Sumai 3' haplotype on 2DL was retained in '13090' and '14006' bred in the 1990s, after 'Saikai 165 ' was introduced as a resistant parent in the Hokkaido lineage (Fig. 5). The composition of the haplotypes on chromosome 2DS in Hokkaido diverged from the haplotypes of the Kyushu lineage (Fig. 5). The 'Gamenya' haplotype was found on chromosome 2DS in old cultivars, 'Timstein', 'Akasabishirazu 1', and 'Norin 8'. Of particular interest were 'Hokuei', 'Kitami 16, 'Kitami 18,' 'Kitami 19', 'Norin 58', and 'Takunekomugi,' which harboured the semi-dwarfing allele of Xgwm261 and 


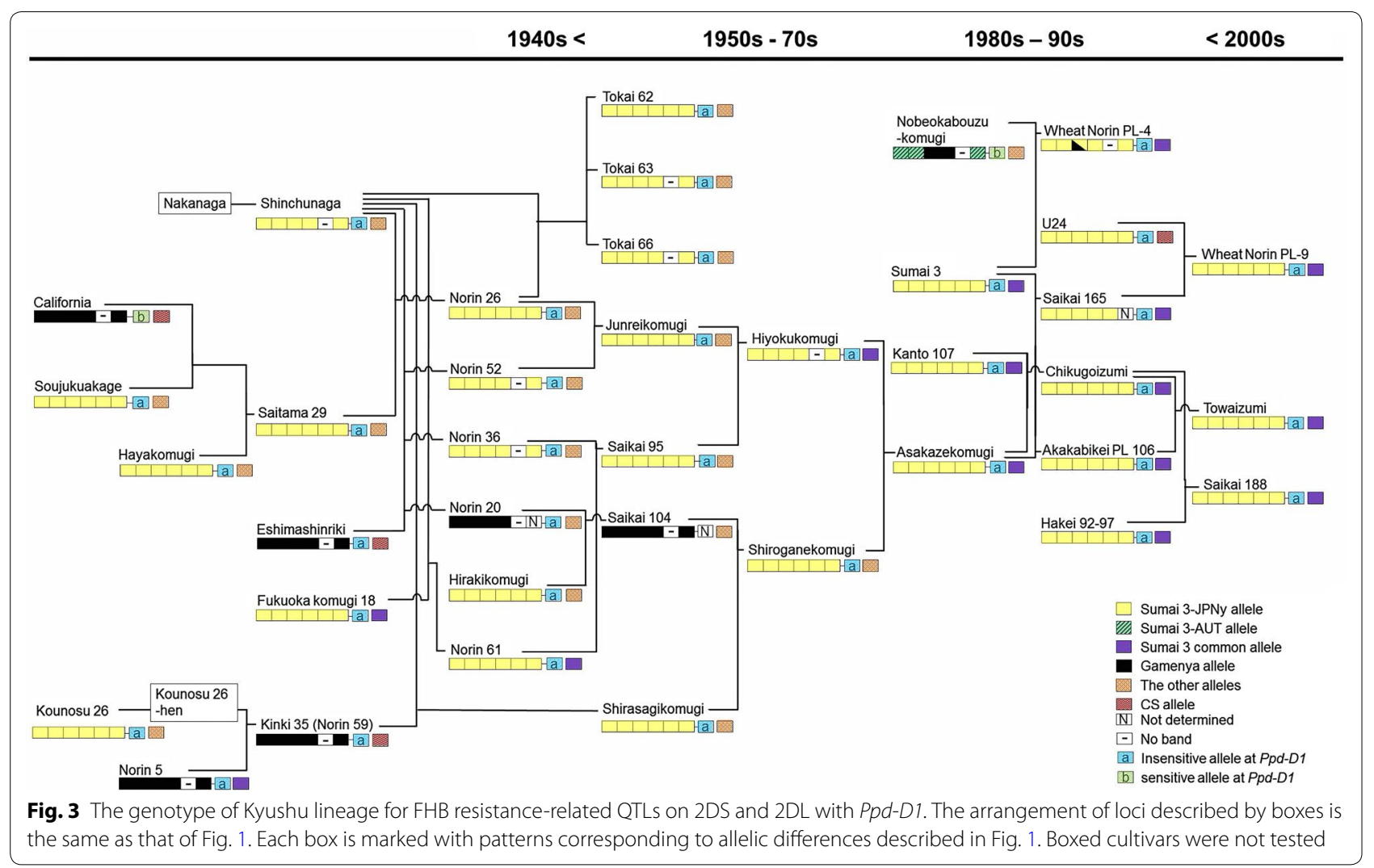

the 'Gamenya' allele of TaMRP-D1 (Fig. 5). The 'Sumai 3-JPNy' haplotype on 2DS from 'Honkei 275' was inherited and retained through 'Hokkai 240', 'Horoshirikomugi', 'Chihokukomugi', and 'Hokushin', and then became fixed in modern cultivars ('Kitamoe,' 'Kitahonami', and '13090') (Fig. 5).

In the spring cultivars of the Hokkaido lineage, the common 'Sumai 3' haplotype on 3BS was found in the lines derived from Kyushu cultivars and its descendants ('Saikai 165,' 'Asakazekomugi,' 'Kitamiharu 764', and 'Kitamiharu 750') (Fig. 4). The 'Sumai 3-JPNy' haplotype on 2DS from 'Saikai 165' was not inherited or detected in its progeny (Fig. 4). The 'Sumai 3-AUT' haplotype on chromosome 2DS (including the resistant 'Gamenya' allele) was derived from two foreign cultivars ('BW 148' and 'Marshall') and in Hokkaido breeding lines ('Kitamiharu 60' and 'Kunkouharu 1047-14') (Fig. 5). The height-promoting allele (165 bp) of Xgwm261 marker associated with Rht8 was retained in all cultivars (Fig. 5).

Although most of the alleles were passed from parents to offspring, several progeny genotypes were distinct. The haplotypes from 9 of 89 cultivars were in a heterozygous state, indicating that some degree of heterozygosity still exists in these lines.

Allelic polymorphism of $P p d-D 1$ was determined in 84 of the 86 cultivars in both lineages (Additional file 3:
Table S3). In the Kyushu lineage, 35 cultivars harboured the insensitive allele $(P p d-D 1 a)$ and two cultivars carried the sensitive allele $(P p d-D 1 b)$ (Additional file 3: Table S3). In the Hokkaido lineage of spring wheat, six cultivars harboured Ppd-D1a and the other six cultivars carried $P p d-D 1 b$ (Additional file 3: Table S3). For winter wheat, 18 cultivars harboured Ppd-D1a and the other 17 cultivars carried $P p d-D 1 b$ (Additional file 3: Table S3).

\section{Discussion}

Difference in haplotype distribution and transmission between the Kyushu and Hokkaido lineages

Analysis of these breeding lineages provided important information regarding the prevalence of FHB resistancerelated QTLs for practical applications. Different haplotype distributions were found between the Kyushu and Hokkaido lineages. In the Kyushu lineage, some resistant haplotypes existed before intensive selection for FHB began in the Japanese national breeding system. The resistant alleles at 3BS, 5AS, and 2DL were retained. By contrast, the old Hokkaido lineage had few haplotypes with resistant alleles. In the Hokkaido lineage, wintertype cultivars lacked resistant haplotypes at 3BS but partially transmitted resistant alleles on 5AS and 2DL in particular pedigrees. The genetic background in the winter wheat includes foreign cultivars that did not undergo 


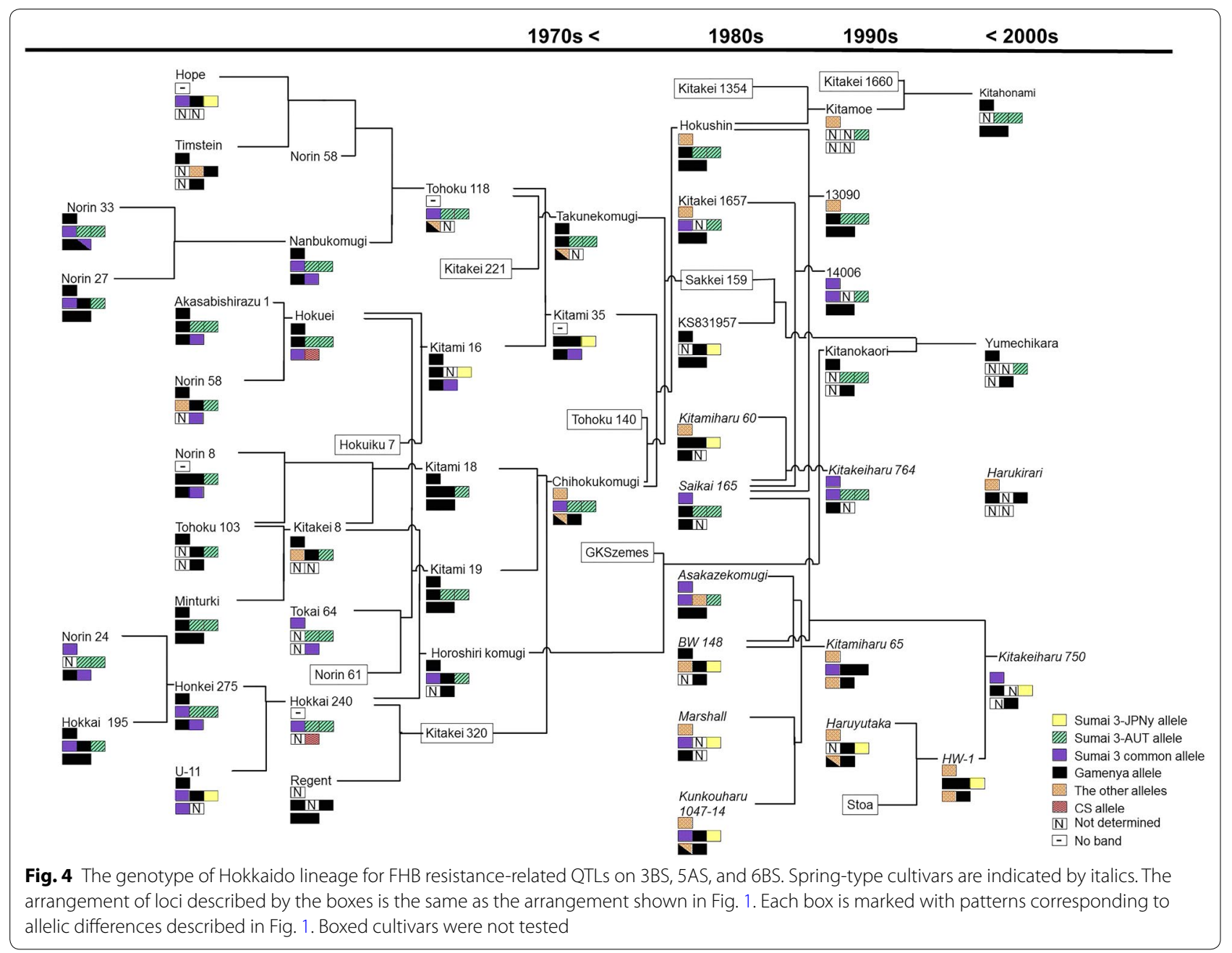

selection for FHB resistance, unlike the southwestern wheat in Japan [28]. This may explain the difference in haplotype distribution between the Kyushu and Hokkaido lineages.

In both lineages, FHB resistance breeding was conducted by crossing resistant cultivars. As the result of the allele introduction and subsequent selection, the current genotypes may be constructed. Therefore, the current results may provide the historical evidence for the important alleles as the results of the allelic selections in the breeding process, in which other agronomic traits as well as FHB resistance have been considered in each cultivation area. This information provides important insight to determine how and what type of alleles should be introduced into current cultivars.

We found that resistant alleles for the FHB QTLs on chromosomes 6BS and 2DS were not always retained in progeny, in which cases alleles related to favourable agronomic traits like for the grain quality in each area might be selected. Such an allelic selection was suggested by data previously. On chromosome 6BS, (1) delayed heading was found on the 'Sumai 3' resistance haplotype on $17.3 \mathrm{cM}$ from Xgwm508 to Xwmc397 in F1-derived doubled-haploid lines developed by crossing 'Kukeiharu 14' (high baking quality) and 'Sumai 3' in Hokkaido [16]. (2) QTLs for quality traits related to starch (peak viscosity, trough viscosity, final viscosity, set back, and peak time) were reported in the cluster region of $17.8 \mathrm{cM}$ between Xgwm644 and Xgwm608b [32]. In addition, previous pedigree analysis identified that pyramiding QTLs for flour yields through ancestors ('Hokushin' and 'Kitamoe', etc.) led to the release of 'Kitahonami' as the highest flour yield cultivar [33]. They also reported that a QTL for flour yield on 6B was common in 'Hokushin' and 'Kitahonami' [33], while both cultivars harboured 'Gamenya' haplotype based on our results (Fig. 4). Similarly, in the case of the Kyushu lineage, the 'Gamenya' haplotype transmitted from 'Saitama 29' on 6B to modern cultivars, 'Wheat Norin PL-9', 'Towaizumi', and 'Saikai 188'. Because 'Saitama 29' was comparable to foreign cultivars in terms 


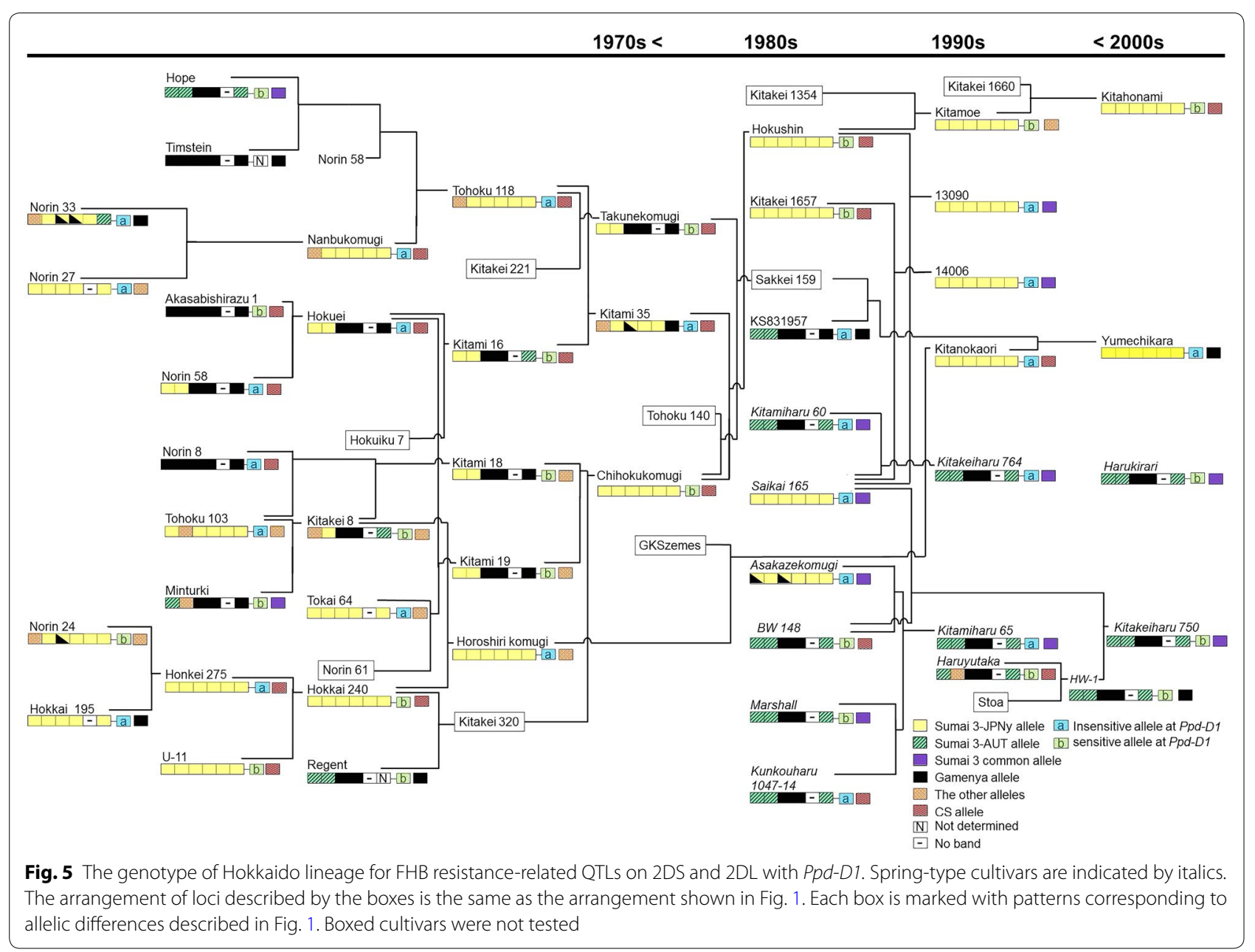

of flour yield, preferential allelic selection for better milling quality might have occurred, and this could explain why 'Gamenya' alleles from 'Saitama 29' retained. The resistant haplotypes on 6BS have been excluded from the Kyushu and the winter type of Hokkaido lineages, potentially due to an association with unfavourable agronomic or quality traits, although we could not exclude the possibility that the 'Gamenya' haplotype is resistant. With respect to chromosome 2DS, the semi-dwarfing allele of Rht8 and the photoperiod-insensitive allele of $P p d-$ D1 were preferably transmitted to Italian wheat cultivars in the beginning of twentieth century [34]. Closely related alleles related to agronomic traits should be taken into account as a favourable allele blocks when the FHB resistance-related QTL on 2DS is introduced.

\section{Conserved haplotypes for FHB QTLs on chromosome 2DS}

The distance between Ppd-D1 and Xgwm261 is enough to produce recombinant haplotypes; TaMRP-D1 is located between Ppd-D1 (20.9 cM from Xgwm261; [35]) and Xgwm261 (6.4 cM from TaMRP-D1; [19]) on 2DS.
Nevertheless, only four haplotypes ('Sumai 3-AUT', 'Sumai 3-JPNy', 'Gamenya, and 'Sumai 3-JPNy' with a null allele on J06F) on 2DS were observed and the 'Sumai $3-J P N y$ ' haplotype was highly conserved in the Kyusyu lineage. This conserved 'Sumai 3-JPNy' haplotype in this region might be explained by alleles underlying agronomic-related characters (Rht8 and Ppd-D1) on 2DS that influence the response to artificial selection.

The geographical distribution of Ppd-D1a alleles in this study corresponded with that of a previous study by Seki et al. [36]. In that study, breeding for early maturity in Japan, except in Hokkaido, was possibly accelerated on the basis of the insensitive allele of Ppd-D1 (Ppd-D1a) [36]. This indicates that the Ppd-D1a allele should be selected because it is a key allele for earliness. Regarding the genotype of Xgwm261 associated with Rht8, a high frequency of cultivars that harboured the semi-dwarfing allele at Xgwm261 was detected in Kyushu cultivars and Hokkaido winter-type cultivars (Table 1). The semidwarfing alleles tend to accumulate, as described previously [37], and Rht1 was found in 'Saitama 29', 'Norin 26', 
Table 1 Genotype of TaMRP-D1 linked by flanked markers of Xgwm261 and Ppd-D1 on chromosome 2DS

\begin{tabular}{|c|c|c|c|c|c|}
\hline Xgwm261 & TaMRP-D1 & Ppd-D1 & Kyushu & Hokkaido spring & Hokkaido winter \\
\hline sd & $\mathrm{S}$ & a & $31(28)$ & $1(1)$ & $10(2)$ \\
\hline sd & $\mathrm{S}$ & $\mathrm{b}$ & 0 & 0 & $7(0)$ \\
\hline sd & $R$ & a & 0 & 0 & $2(0)$ \\
\hline $\mathrm{sd}$ & $\mathrm{R}$ & $\mathrm{b}$ & 0 & 0 & $4(0)$ \\
\hline ne & $\mathrm{S}$ & a & 0 & 0 & 0 \\
\hline ne & $\mathrm{S}$ & $\mathrm{b}$ & 0 & 0 & 0 \\
\hline ne & $R$ & a & $4(1)$ & 0 & $1(0)$ \\
\hline ne & $R$ & $b$ & $1(0)$ & 0 & $1(0)$ \\
\hline hp & $\mathrm{S}$ & a & 0 & 0 & 0 \\
\hline hp & $\mathrm{S}$ & $\mathrm{b}$ & 0 & 0 & 0 \\
\hline hp & $R$ & a & 0 & $4(1)$ & $1(0)$ \\
\hline hp & $\mathrm{R}$ & $b$ & $1(1)$ & $6(1)$ & $3(0)$ \\
\hline Number of cultivars & & & 37 & 11 & 29 \\
\hline
\end{tabular}

sd, semi-dwarf (Sumai 3-JPNy and Chinese Spring allele); ne, neutral phenotype (Gamenya allele); hp, height promotion (Sumai 3-AUT and Nobeokabouzu-komugi allele); $S$, susceptible; $R$, resistant; $a$, insensitive; $b$, sensitive. A number within brackets mean a number of cultivars which harboured resistant allele at $3 B S$

'Junreikomugi', 'Shirasagikomugi', 'Hiyokukomugi,' 'Shiroganekomugi', and 'Asakazekomugi', as well as Rht2 in 'Hayakomugi' and 'Norin 61' in the surveyed lines of the Kyushu lineage. The high frequency of the semi-dwarfing allele at $X g w m 261$ linked to Rht8 implies a role for dwarfism associated with major $R h t$ genes. It is also likely that preferential selection of dwarfing genes played a role in lowering FHB resistance level, because $R h t 1$ and $R h t 2$ are known to be associated with FHB susceptibility (type I) [38].

The conservation of the 'Sumai 3-JPNy' haplotype may be attributed to the selective advantage described in Pestsova and Röder [39]. Both the semi-dwarfing Rht8 allele and the photoperiod-insensitive $P p d-D 1$ allele not only cause early flowering, but also reduce plant height, resulting in increased wheat yield and a selective advantage [34]. Based on the breeding objectives in Kyushu (i.e. earliness), the high frequency of conserved linkage between the semi-dwarfing allele of Xgwm261 and the insensitive allele of Ppd-D1 (Ppd-D1a) might be expected (Table 1). Based on the present observations in the Kyushu lineage, the conservation of the 'Sumai 3-JPNy' haplotype is hypothesized to have resulted from the fixation of the semi-dwarfing allele of Xgwm261 and the insensitive allele of Ppd-D1 (Ppd-D1a).

However, in some cultivars of the Hokkaido lineage, the 'Sumai 3-JPNy' haplotype was conserved, independent of the Ppd-D1 genotype; the sensitive allele of Ppd-D1 (Ppd$D 1 b)$ was frequently found in the winter-type cultivars, which carried the susceptible TaMRP-D1 allele on chromosome 2DS (Table 1, Fig. 5). The insensitive allele of Ppd-D1 (Ppd-D1a) may not confer a fitness advantage in Hokkaido. Irrespective of the $P p d-D 1$ allele, linkage was conserved between the semi-dwarfing or height promotion/neutral phenotype alleles of Xgwm261 and susceptible or resistant alleles of TaMRP-D1 (Table 1). Yamada [37] also found $R h t 1$ in 'Haruyutaka' spring-type plants, as well as Rht2 in 'Norin 24,' 'Norin 33,' 'Norin 58,' 'Nanbukomugi, 'Takunekomugi', and 'Horoshirikomugi' from winter-type lines of the Hokkaido lineage, suggesting that the semi-dwarf phenotype has been a major breeding objective in Hokkaido. Thus, it is possible that $R h t 8$ plays a role in dwarfism with $R h t 1$ or $R h t 2$ in Hokkaido. Taken together, positive selection of $P p d-D 1 a$ is not always needed for the conservation of the 'Sumai 3-JPNy' haplotype. To find other causal alleles for this conservation, further studies on 2DS will be needed.

The conservation of the 'Sumai 3-JPNy' haplotype is expected to weaken $\mathrm{FHB}$ resistance, due to the effect of the susceptible allele of TaMRP-D1. This is thought to be inconsistent with the process of FHB resistance selection in the Kyushu lineage. Our results provided important insight regarding this argument; 15 of the 23 cultivars with the 'Sumai 3-JPNy' haplotype harboured more than two FHB resistance-related QTL alleles (Additional file 4: Table S4). Another five cultivars with zero or one FHB resistance-related QTL alleles were Chinese strain (' $U$ 24') or cultivars bred before the initiation of FHB-resistant breeding ('Saitama 29,' 'Norin 26,' 'Shirasagikomugi', and 'Kounosu 26'), indicating the main breeding targets were less strongly selected than FHB resistance. In the 1930s-1940s, the resistant alleles on 6BS were transmitted to several generations and then were replaced by the 'Gamenya' haplotypes (Fig. 2). In contrast, the resistant allele on 2DL was introduced in the 1970s and has been retained (Fig. 2). The resistant haplotypes on 3BS 
and 5AS were also frequently harboured by the cultivars bred after the 1970s. Based on the accumulation of FHB resistance-related QTL alleles on other chromosomes, the 'Sumai 3-JPNy' haplotype on 2DS could be retained during FHB resistance selection, allowing the development of cultivars exhibiting both semi-dwarfing (derived from $R h t 8$ ) and FHB resistance phenotypes (derived from $3 \mathrm{BS}, 5 \mathrm{AS}$, and $6 \mathrm{BS}$ ). A similar argument may apply to the conservation of the resistant alleles on 5AS. The resistant alleles ('Sumai 3' alleles) on 5AS are in close proximity to the QTL for increased stem length [16]. The resistant haplotypes on 5AS might be allowed under a genetic background that includes $R h t$ genes (see above). It is likely that a trade-off between FHB-resistant-related QTL alleles and those related to other agronomic traits has contributed to the history of FHB resistance breeding.

\section{FHB resistance corresponded with the allelic genotypes of TaMRP-D1}

The genotypes of representative alleles on 2DS, 3BS, 5AS, and $6 \mathrm{BS}$ were considered together with the FHB resistance phenotype, assessed by evaluation in the greenhouse and in the field, as reported in Ban and Suenaga [23] (Additional file 5: Table S5). Alleles on 2DL were not included owing to the large number of plants with an undetermined resistant type. 'Nobeokabouzu-komugi', known as a highly resistant landrace, had resistant alleles at five loci and was assessed as very resistant (VR), while cultivars carrying the susceptible allele at TaMRP-D1 were assessed as less than VR (ranging between very resistant-resistant (VR-R) and susceptible (S)) (Additional file 5: Table S5). Because the resistant allele of Fhb1 is known to have a great effect on the total phenotypic variation of FHB resistance (up to $60 \%$, as reviewed in Buerstmayr et al. [4]), at least a moderate level of FHB resistance may be expected, if a cultivar carries the resistant allele at this locus. The effect of each QTL on FHB resistance has been well demonstrated in numerous previous studies (reviewed in Buerstmayr et al. [4]). Our genotyping data showed a correlation with previous data regarding QTL effects in Kyushu cultivars. Therefore, the resistant 'Gamenya' allele of TaMRP-D1 on 2DS may have a positive effect on FHB resistance. While the phenotypic variance of FHB resistance was $8.1-24.1 \%$ on 5 AS $[9,10]$ and $2.9-4.4 \%$ on 6 BS [14], that of FHB resistance was $14-25 \%$ on 2DS [19]. This effect could be expected if the 'Sumai 3-JPNy' allele was replaced with the 'Gamenya' allele. Niwa et al. [29] also detected the resistance of genotypes that harboured the 'Gamenya' allele of TaMRP-D1 on 2DS in different 'Sumai 3' accessions.

Based on our results, we proposed that a genotype that has a resistant haplotype on 2DS might achieve further enhancement of FHB resistance in modern cultivars that harbour resistant ones at 2DL, 3BS, and 5AS. On chromosome 2DS, winter wheat cultivars harbouring the resistant 'Gamenya' allele of TaMRP-D1 accompanied by the semi-dwarfing 'Sumai 3-JPNy' allele of Xgwm261 were particularly valuable for introducing FHB resistance alleles without introducing the height-promoting allele of Xgwm261. Therefore, recombinant cultivars 'Hokuei', 'Kitami 16,' 'Kitami 18,' 'Kitami 19', 'Norin 58', and 'Takunekomugi' can be useful materials as donors of FHB resistance alleles of FHB-QTL on 2DS, because they can introduce both the 'Gamenya' and 'Sumai 3-JPNy' haplotypes on 2DS into modern cultivars ('Norin PL-9', 'Saikai 188,' 'Towaizumi', 'Kitahonami', and 'Yumechikara'). Otherwise, the linkage block of the resistant 'Gamenya' allele of TaMRP-D1 and the alleles associated with height promotion/neutral phenotypes at Xgwm261 on chromosome 2DS can be unlinked using DNA marker selection when introducing the gene block of TaMRP-D1 and Xgwm261. The genetic distance between them is sufficiently long to obtain a recombinant gene block. Using this strategy, the haplotypes on 2DS can be replaced to increase FHB resistance in modern cultivars (e.g. 'Norin PL-9', 'Saikai 188,' 'Towaizumi', 'Kitahonami', and 'Yumechikara') that carry the susceptible TaMRP-D1 allele.

\section{Conclusions}

The subsequent allelic selection on chromosomes 2DS and 6BS was common in Kyushu and Hokkaido lineages, indicating that trade-off between FHB resistance and other agronomic traits has contributed to the history of wheat breeding. To enhance FHB resistance in current cultivars, the resistant allele of QTL on 2DS can be introduced. We also found the six cultivars having both FHBresistant allele of TaMRP-D1 and semi-dwarfing allele of Xgwm261. These cultivars can be used as crossing parents to achieve further enhancement of FHB resistance in modern cultivars while maintaining their semi-dwarf trait.

\section{Additional files}

Additional file 1: Table S1. Origins and type of wheat cultivars used for the genotyping.

Additional file 2: Table S2. DNA markers associated with FHB-resistant QTL derived from Sumai 3 in this study.

Additional file 3: Table S3. Ppd-D1 genotype of the Kyushu and Hokkaido wheat lineages.

Additional file 4: Table S4. Genotypes of FHB resistance-related QTL alleles on 3BS, 5AS, and 2DL in cultivars having Sumai 3-JPNy haplotype.

Additional file 5: Table S5. Representative results of genotypes for Sumai 3-related QTLs in Kyushu wheat lines with reported FHB evaluation. 


\section{Abbreviations}

FHB: Fusarium head blight; QTLs: quantitative trait loci; DON: deoxynivalenol; CS: Chinese Spring.

\section{Authors' contributions}

All authors contributed to manuscript improvement. All authors read and approved the final manuscript.

\section{Author details}

${ }^{1}$ Kihara Institute for Biological Research, Yokohama City University, 641-12 Maioka, Totsuka, Yokohama, Kanagawa 244-0813, Japan. ${ }^{2}$ RIKEN Nishina Center, 2-1 Hirosawa, Wako, Saitama 351-0198, Japan.

\section{Acknowledgements}

The authors are grateful to the National Agriculture and Food Research Organization (NARO)/Kyushu Okinawa Agricultural Research Center, the NARO/ Hokkaido Agricultural Research Center, the National Institute of Agrobiological Sciences, and the Hokkaido Research Organization/Kitami Agricultural Experiment Station for providing seeds. The authors appreciate the RIKEN Research Resources Center of the Brain Science Institute for performing DNA sequencing.

\section{Competing interests}

The authors declare that they have no competing interests.

\section{Availability of data and materials}

The data sets generated and analysed during the current study are available from the corresponding author on reasonable request.

\section{Consent for publication}

Not applicable.

\section{Ethics approval and consent to participate}

Not applicable.

\section{Funding}

This work was supported in part by a grant from the Ministry of Agriculture, Forestry and Fisheries of Japan (MAFF) (Genomics for Agricultural Innovation, TRG-1005).

\section{Publisher's Note}

Springer Nature remains neutral with regard to jurisdictional claims in published maps and institutional affiliations.

Received: 21 December 2016 Accepted: 3 October 2017

Published online: 09 January 2018

\section{References}

1. Van Egmond HP, Jonker MA. Worldwide regulations for mycotoxins in food and feed in 2003. Food and Agriculture Organization of the United Nation: Rome, Italy. 2004. http://www.fao.org/docrep/007/y5499e/ y5499e00.htm. Accessed on 15 Sept 2016.

2. Gilbert J, Tekauz A. Strategies for management of Fusarium head blight (FHB) in cereals. Prairie Soils Crops. 2011;4:97-104.

3. Schroeder HW, Christensen JJ. Factors affecting resistance of wheat to scab caused by Gibberella zeae. Phytopathology. 1963;53:831-8.

4. Buerstmayr H, Ban T, Anderson JA. QTL mapping and marker-assisted selection for Fusarium head blight resistance in wheat; a review. Plant Breed. 2009;128:1-26.

5. Holzapfel JH, Voss H, Miedaner T, Korzun V, Häberle J, Schweizer G, Mohler V, Zimmermann G, Hartl L. Inheritance of resistance to Fusarium head blight in three European winter wheat populations. Theor Appl Genet. 2008;117:1119-28.

6. Bai G, Shaner G. Scab of wheat: prospects for control. Plant Dis. 1994;78:760-6.

7. Bai G, Shaner G. Management and resistance in wheat and barley to Fusarium head blight. Annu Rev Phytopathol. 2004;42:135-61.
8. Anderson JA, Stack RW, Liu S, Waldron BL, Fjeld AD, Coyne C, MorenoSevilla B, Fetch JM, Song QJ, Cregan PB, et al. DNA markers for Fusarium head blight resistance QTLs in two wheat populations. Theor Appl Genet. 2001;102:1164-8.

9. Buerstmayr H, Lemmens M, Hartl L, Doldi L, Steiner B, Stierschneider M, Ruckenbauer P. Molecular mapping of QTLs for Fusarium head blight resistance in spring wheat. I. Resistance to fungal spread (Type II resistance). Theor Appl Genet. 2002;104:84-91.

10. Buerstmayr $\mathrm{H}$, Steiner B, Hartl L, Griesser M, Angerer N, Lengauer D, Miedaner T, Schneider B, Lemmens M. Molecular mapping of QTLs for Fusarium head blight resistance in spring wheat. II. Resistance to fungal penetration and spread. Theor Appl Genet. 2003;107:503-8.

11. Cuthbert PA, Somers DJ, Thomas J, Cloutier S, Brûlé-Babel A. Fine mapping Fhb1, a major gene controlling Fusarium head blight resistance in bread wheat (Triticum aestivum L.). Theor Appl Genet. 2006;112:1465-72.

12. Kollers S, Rodemann B, Ling J, Korzun V, Ebmeyer E, Argillier O, Hinze M, Plieske J, Kulosa D, Ganal MW, Röder MS. Whole genome association mapping of Fusarium head blight resistance in European winter wheat (Triticum aestivum L.). PLoS ONE. 2013;8:e57500.

13. Liu S, Pumpherey MO, Gill BS, Trick HN, Zhang JX, Dolezel J, Chalhoub B, Anderson JA. Toward positional cloning of Fhb1, a major QTL for Fusarium head blight resistance in wheat. Cereal Res Commun. 2008;36:195-201.

14. Shen X, Zhou M, Lu W, Ohm H. Detection of Fusarium head blight resistance QTL in a wheat population using bulked segregant analysis. Theor Appl Genet. 2003;106:1041-7.

15. Cuthbert PA, Somers DJ, Brulé-Babel A. Mapping of Fhb2 on chromosome 6BS: a gene controlling Fusarium head blight field resistance in bread wheat (Triticum aestivum L.). Theor Appl Genet. 2007;114:429-37.

16. Suzuki T, Sato M, Takeuchi T. Evaluation of the effects of five QTL regions on Fusarium head blight resistance and agronomic traits in spring wheat (Triticum aestivum L.). Breed Sci. 2012;62:11-7.

17. Rawat N, Pumphrey MO, Liu S, Zhang X, Tiwari VK, Ando K, Trick HN, Bockus WW, Akhunov E, Anderson JA, Gill BS. Wheat Fhb1 encodes a chimeric lectin with agglutinin domains and a pore-forming toxin-like domain conferring resistance to Fusarium head blight. Nat Genet. 2016. doi:10.1038/ng.3706.

18. Basnet RB, Glover KD, Ibrahim AMH, Yen Y, Chao S. A QTL on chromosome 2DS of 'Sumai 3 'increases susceptibility to Fusarium head blight in wheat. Euphytica. 2012;186:91-101.

19. Handa H, Namiki N, Xu D, Ban T. Dissecting of the FHB resistance QTL on the short arm of wheat chromosome 2D using a comparative genomic approach: from QTL to candidate gene. Mol Breed. 2008;22:71-84.

20. Ban T. Physiology and ecology of Fusarium head blight in Triticeae, and wheat resistance. (Mugirui akakabibyou no seiri seitai oyobi komugi no teikousei) Shokubutsu Boeki-Plant Protection. 2002;56:58-63. http://www.jppa.or.jp/shuppan/images-txt/2002/2002_0205.pdf (in Japanese)

21. Gocho H, Hirai T, Kashiwao T. Fusarium head blight resistance in Chinese wheat varieties, Sumai 2 and Sumai 3. Kyushu nogyo kenkyu. 1978;40:3132. http://www.naro.affrc.go.jp/org/karc/qnoken/qnoken/no40/40-031. pdf (in Japanese)

22. Gocho H, Hirai T, Kashiwao T. Breeding of Wheat Norin PL-4 a new germplasm with resistance to scab (Gibberella zeae (Schw.) Petch). Bulletin of the National Agricultural Research Center for Kyushu Okinawa Region. 1992;27:317-31. http://agriknowledge.affrc.go.jp/RN/2010461726.pdf (in Japanese)

23. Ban T, Suenaga K. Genetic analysis of resistance to Fusarium head blight caused by Fusarium graminearum in Chinese wheat cultivar Sumai 3 and the Japanese cultivar Saikai 165. Euphytica. 2000;113:87-99.

24. Kubo K, Kawada N, Fujita M, Hatta K, Matsunaka H, Oda S, Hatano T, Seki M, Yoshioka T, Otobe C, Nakajima T. Development of 'Wheat Norin-PL 9 (Akakabikei 3)', a cleistogamous wheat line with Fusarium head blight resistance. Bulletin of the National Agricultural Research Center for Kyushu Okinawa Region. 2012;57:21-34. http://agriknowledge.affrc.go.jp/ RN/2010831013.pdf (in Japanese).

25. Oda S, Kawada N, Fujita M, Hatta K, Ujihara K, Sasaki A, Taya S, Tsutsumi T, Taniguchi Y, Seki M, Tohnooka T, Taira M, Hatano T, Ban T. New wheat cultivar "Towaizumi". Bulletin of the National Agricultural Research Center for Kyushu Okinawa Region. 2009;51:19-39. http://agriknowledge.affrc. go.jp/RN/2010772284.pdf (in Japanese). 
26. Kubo K, Kawada N. Varietal difference in resistance to spread of Fusarium head blight and its relation with grain mycotoxin accumulation in western Japanese wheat. Breed Sci. 2009;59:261-8.

27. Yanagisawa A. Progress in improvement of Fusarium head blight resistant wheat varieties with low mycotoxin accumulation. JSM Myctoxins. 2006;56:17-23. https://www.jstage.jst.go.jp/article/myco/56/1/56_1_17/_ pdf (in Japanese).

28. Nishio Z, Takata K, Tabiki T, Ito M, Takenaka S, Kuwabara T, Iriki N, Ban T. Diversity of resistance to Fusarium head blight in Japanese winter wheat. Breed Sci. 2004;54:79-84.

29. Niwa S, Kubo K, Lewis J, Kikuchi R, Alagu M, Ban T. Variations for Fusarium head blight resistance associated with genomic diversity in different sources of the resistant wheat cultivar 'Sumai 3'. Breed Sci. 2014;64:90-6.

30. Worland AJ, Korzun V, Röder MS, Ganal MW, Law CN. Genetic analysis of the dwarfing gene Rht8 in wheat. Part II. The distribution and adaptive significance of allelic variants at the Rht 8 locus of wheat as revealed by microsatellite screening. Theor Appl Genet. 1998;96:1110-20.

31. Beales J, Turner A, Griffiths S, Snape JW, Laurie DA. A Pseudo-Response Regulator is misexpressed in the photoperiod insensitive Ppd-Dla mutant of wheat (Triticum aestivum L.). Theor Appl Genet. 2007;115:721-33.

32. Sun H, Lu J, Fan Y, Zhao Y, Kong F, Li R, Wang H, Li S. Quantitative trait loci (QTLs) for quality traits related to protein and starch in wheat. Prog Nat Sci. 2008;18:825-31.
33. Ishikawa G, Nakamura K, Ito H, Saito M, Sato M, Jinno H, Yoshimura Y, Nishimura T, Maejima H, Uehara Y, Kobayashi F, Nakamura T. Association mapping and validation of QTLs for flour yield in the soft winter wheat variety Kitahonami. PLoS ONE. 2014;9:e111337.

34. Borojevic K, Borojevic K. The transfer and history of "reduced height genes" (Rht) in wheat from Japan to Europe. J Hered. 2005;96:455-9.

35. Chebotar GO, Chebotar SV, Motsnyy II, Sivolap YM. Clarification of the Rht8-Ppd-D1 gene linkage on the 2D chromosome of winter bread wheat. Cytol Genet. 2013;47:70-4.

36. Seki M, Chono M, Matsunaka H, Fujita M, Oda S, Kubo K, Kiribuchi-Otobe C, Kojima H, Nishida H, Kato K. Distribution of photoperiod-insensitive alleles Ppd-B1a and Ppd-D1 $a$ and their effect on heading time in Japanese wheat cultivars. Breed Sci. 2011;61:405-12.

37. Yamada T. Classification of GA response, Rht genes and culm length in Japanese varieties and landraces of wheat. Euphytica. 1990;50:221-39.

38. He X, Singh KP, Dreisigacker S, Singh S, Lillemo M, Duveiller E. Dwarfing genes Rht-B1b and Rht-D1b are associated with both type I FHB susceptibility and low anther extrusion in two bread wheat populations. PLoS ONE. 2016. doi:10.1371/journal.pone.0162499.

39. Pestsova E, Röder M. Microsatellite analysis of wheat chromosome 2D allows the reconstruction of chromosomal inheritance in pedigrees of breeding programmes. Theor Appl Genet. 2002;106:84-91.

\section{Submit your next manuscript to BioMed Central and we will help you at every step:}

- We accept pre-submission inquiries

- Our selector tool helps you to find the most relevant journal

- We provide round the clock customer support

- Convenient online submission

- Thorough peer review

- Inclusion in PubMed and all major indexing services

- Maximum visibility for your research

Submit your manuscript at www.biomedcentral.com/submit 